\title{
Switching stable patients with schizophrenia from their oral antipsychotics to aripiprazole lauroxil: a post hoc safety analysis of the initial 12-week crossover period
}

\author{
Peter J. Weiden, ${ }^{\prime *}$ Yangchun Du, ${ }^{2}$ Chih-Chin Liu, ${ }^{2}$ and Arielle D. Stanford ${ }^{3}$
}

\footnotetext{
${ }^{1}$ Medical Affairs, Alkermes, Inc., Waltham, Massachusetts, USA

${ }^{2}$ Clinical Development, Alkermes, Inc., Waltham, Massachusetts, USA

${ }^{3}$ Clinical Science, Alkermes, Inc., Waltham, Massachusetts, USA
}

Objective. Switching antipsychotic medications is common in patients with schizophrenia who are experiencing persistent symptoms or tolerability issues associated with their current drug regimen. This analysis assessed the safety of switching from an oral antipsychotic to the long-acting injectable antipsychotic aripiprazole lauroxil (AL).

Methods. This was a post hoc analysis of outpatients with schizophrenia who were prescribed an oral antipsychotic and who enrolled in an international, open-label, long-term (52-week) safety study of AL. The analysis focused on the first 3 injections of AL $882 \mathrm{mg}$ over 12 weeks, divided into the immediate 4-week crossover period between the first and second AL injections (initiation phase) and the subsequent 8 weeks (stabilization phase). Patients were grouped by preswitch oral antipsychotic medication, and safety and clinical symptoms were assessed.

Results. In total, 190 patients had switched from one of the following oral antipsychotic medications: aripiprazole, conventional antipsychotics, risperidone/paliperidone, olanzapine, or quetiapine. The 12-week completion rate was high $(92.1 \%)$ and similar across the different preswitch oral antipsychotic groups. Overall, adverse event (AE) rates experienced over 12 weeks were modest; no AEs were considered serious. The most common AEs in the initiation phase were injection site pain $\mathbf{5 . 8 \%}$ ), insomnia $(5.8 \%)$, and akathisia (3.2\%). No apparent relationship was observed between preswitch medication and early-onset AEs. Mean Positive and Negative Syndrome Scale total scores remained stable during this period across preswitch antipsychotic groups.

Conclusion. Switching from an oral antipsychotic to AL was feasible in an outpatient setting for patients with schizophrenia, and the 12-week retention rate was favorable.

Received 8 January 2018; Accepted 17 April 2018; First published online 26 June 2018

Key words: Long-acting antipsychotics, medication switch, oral antipsychotics, safety, schizophrenia, tolerability.

* Address for correspondence: Peter J. Weiden, MD, Schizophrenia Lead, Medical Affairs, Alkermes, Inc., 852 Winter Street, Waltham, MA 02451-1420, USA.

*(Email: Peter.Weiden@alkermes.com)

This study was sponsored by Alkermes, Inc., Waltham, MA, USA. Funding for editorial support was provided by Alkermes, Inc., Waltham, MA, USA. The authors thank all the patients and investigators who participated in and contributed to this study. The authors also thank Dr Robert Risinger (formerly of Alkermes, Inc.) for their contribution to the analyses of the study. Medical writing and editorial support for the preparation of this manuscript (under the guidance of the authors) was provided by Karen Yee, PhD (ApotheCom, UK).

ClinicalTrials.gov identifier: NCT01626456; EudraCT Number: 2012 003996-20.

\section{Introduction}

Pharmacotherapy has long been a cornerstone in the long-term management of schizophrenia, with both oral and long-acting injectable (LAI) antipsychotic medications. ${ }^{1}$ However, persistent symptoms, tolerability issues, and the nature of the disease itself (poor insight and cognitive deficits) can make it difficult for patients to adhere to a daily treatment regimen. ${ }^{2,3}$ Even small gaps in treatment can double the risk for hospitalization. ${ }^{4}$

Switching of antipsychotic medication is often considered for patients with persistent symptoms or tolerability issues during maintenance management of schizophrenia. ${ }^{1,5}$ 
Although antipsychotics are available as oral or LAI formulations, oral medication is prescribed to most outpatients; LAIs are commonly reserved for nonadherent or "revolving door" patients. ${ }^{6}$ Oral antipsychotics are also usually the medication of choice when switching antipsychotics. However, clinicians experienced with switching from one oral to another oral antipsychotic might be hesitant to recommend switching to a long-acting antipsychotic because of concerns about the ease and safety of making the switch. ${ }^{7}$ Common concerns can include the likelihood and seriousness of any new adverse events (AEs) that occur during the crossover process, whether these problems are transient or persistent, and the likelihood that the patient will continue taking the LAI after the initial switching period. ${ }^{7}$

Aripiprazole lauroxil (AL) is a prodrug of aripiprazole that was developed as a long-acting intramuscular formulation for the treatment of schizophrenia. ${ }^{8}$ The efficacy and safety of AL compared with placebo was established in a 12-week, randomized, double-blind study for the treatment of patients with acute exacerbation of schizophrenia. ${ }^{9}$

Herein, we report the findings of a post hoc analysis of a 52-week open-label study conducted to assess the longterm effects of monthly AL treatment in patients with stable schizophrenia. The overall safety results of the 52-week study will be presented elsewhere (Nasrallah et al., 2018). The post hoc analysis will focus on safety outcomes during the initial transition period (corresponding to the first 12 weeks of the study) in patients who have transitioned from their current oral antipsychotic to AL.

\section{Methods}

A 52-week, open-label, multicenter, safety study of AL (ClinicalTrials.gov identifier: NCT01626456) was conducted to assess the long-term safety and durability of therapeutic effect of monthly AL treatment in patients with stable schizophrenia. Participants in this study included patients who had successfully completed the initial 12-week efficacy study ${ }^{9}$ and outpatients with schizophrenia who were switching from their current oral antipsychotic to AL (Figure S1, available online in the supplemental material). Herein, we report the results of a post hoc analysis focusing on safety outcomes during the initial transition period (covering the first $3 \mathrm{AL}$ injections) in patients who switched from their current oral antipsychotic to AL.

\section{Study design}

After screening and enrollment, these patients received their first dose of AL (882 mg), a dose that has been modeled to be equivalent to an oral dose of aripiprazole $20 \mathrm{mg} /$ day, ${ }^{11}$ while their current oral antipsychotic was tapered (Figure 1). These patients continued to receive $\mathrm{AL} 882 \mathrm{mg}$ every 4 weeks thereafter for the next 52 weeks (Figure S1).

The current post hoc analysis focused on the first 12 weeks of treatment in a subset of these patients after the first injection of $\mathrm{AL}$ because this period of change in medication regimen can create new tolerability issues, in addition to uncertainty about the relative effectiveness of the new, postswitch agent. The 12-week period was further divided into 2 phases, the initiation and stabilization phases (Figure 1), based on the treatments that the patient received. The initiation phase (4 weeks) consisted of the time between the first AL injection until the time the patient returned for his or her second injection approximately 4 weeks later. The stabilization phase ( 8 weeks) covered the period from the second $\mathrm{AL}$ injection to just before the fourth injection at or around 12 weeks after the first AL injection (Figure 1). Details of

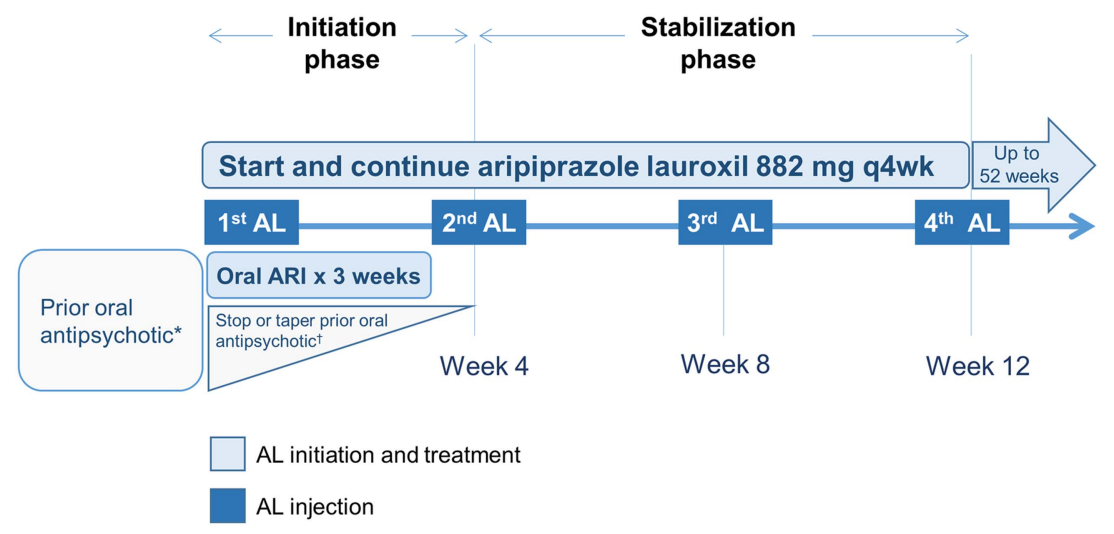

FIGURE 1. Overview of switching from an oral antipsychotic to aripiprazole lauroxil. *Patients not previously exposed to aripiprazole were assessed for tolerability to aripiprazole during the screening phase. ${ }^{\dagger}$ For patients taking oral antipsychotics (other than aripiprazole), treatment could be stopped or a crossover down-titration could be initiated over the next 4 weeks, as per investigator judgment (concurrently with the 3 weeks of daily 15 mg oral aripiprazole). $\mathrm{AL}$, aripiprazole lauroxil; ARI, aripiprazole. 
the specific steps taken to switch from the oral medication are provided below.

The study was conducted in accordance with the Declaration of Helsinki, and Good Clinical Practice Guidelines by the International Conference on Harmonization 1997. The study protocol, amendments, and informed consent forms were approved by an independent ethics committee/institutional review board for each site. All patients provided written informed consent before entering the study.

\section{Patients}

Key inclusion criteria for outpatients who were taking an oral antipsychotic at the time they entered the 52-week AL safety study included a diagnosis of schizophrenia, clinically stable status, having been prescribed the same dose of a first-line oral antipsychotic for the 2 months before enrollment, not having active symptoms (as defined by a Clinical Global Improvement for Severity [CGI-S] subscale $\leq 3$ [mild] and Positive and Negative Symptom Scale [PANSS] total score $<70$ ), and potentially benefiting from initiation of AL.

Patients were included in the current post hoc analysis if they could be grouped in specific preswitch oral antipsychotic groups. Two of the authors (CCL and PJW) reviewed prior antipsychotic medication use in the case report form to identify cohorts of different preswitch oral antipsychotics to form a basis of group comparisons. A minimum number of 19 patients was necessary to qualify as a preswitch oral group. Antipsychotics taken by fewer than 19 patients and, thus, not included in this post hoc analysis are summarized in Table S1 (available online in the Supplemental Material).

Key exclusion criteria included history of nonresponse or intolerance to oral aripiprazole, recent treatment with any long-acting antipsychotic formulation, history of treatment resistance (eg, prior treatment with clozapine), the presence of any medically significant comorbidity, active substance comorbidity, or at high risk of causing harm to self or others.

\section{Switching strategy from oral antipsychotics to AL}

During the screening period, patients who were taking an oral antipsychotic other than aripiprazole were assessed for tolerability to aripiprazole. Those without prior exposure to aripiprazole received test doses of $5 \mathrm{mg}$ oral aripiprazole for 2 days before starting the initiation phase.

As part of the initiation phase, patients received a first AL 882-mg injection, along with a 3-week supply of oral aripiprazole 15-mg tablets for daily dosing, after which oral aripiprazole was discontinued (Figure 1). For patients who had been receiving oral aripiprazole as a preswitch medication, the prescribed tablets were discontinued and the patient was instructed to use the 3 -week supply provided in the study. The preswitch aripiprazole group did not change oral antipsychotics, but dose changes were possible. Preswitch (nonaripiprazole) oral antipsychotics could be continued or a crossover down-titration could be initiated over the next 4 weeks, as per investigator judgment. In the stabilization phase, the second $\mathrm{AL}$ 882-mg injection was administered 4 weeks after the first; no oral antipsychotic was allowed after this visit. Patients returned every 4 weeks thereafter for assessment and AL injections. Patients were allowed to use benzodiazepines for anxiety and/or insomnia, and anticholinergics and other standard adjunctive treatments for new or worsening extrapyramidal symptoms or akathisia.

\section{Study assessments}

All assessments reported herein are for the 12-week period between the first AL injection until the fourth injection visit. The key outcomes in this post hoc analysis were study retention, as defined by the proportion of patients who received their second $\mathrm{AL}$ injection and successfully completed the initial crossover (initiation phase retention), and the proportion of patients who received the first $3 \mathrm{AL}$ injections over 12 weeks (initiation/stabilization phase retention).

Early-onset AEs were recorded and categorized by their period of occurrence, either during the initiation phase or the stabilization phase, grouped by preswitch antipsychotic. AEs of interest included extrapyramidal symptoms, akathisia, and other AEs commonly associated with changing antipsychotics (eg, disruption in sleep-wake cycle) or starting an LAI medication (eg, injection site reactions). Other safety variables of interest that have been reported as sensitive to the preswitch antipsychotic $^{12}$ were also evaluated; these included changes in weight, body mass index, and serum prolactin levels between baseline and week 12 .

Efficacy was assessed using changes from baseline in PANSS total and CGI-S scale scores, as well as relapse rates. Patients were considered to have experienced a relapse or to be expecting an impending relapse if either of the following criteria were met: (1) hospitalization or emergency room visit or (2) a Clinical Global Impression for Improvement (CGI-I) score of 5 (minimally worse, much worse, or very much worse), and one of the following: (a) PANSS item P2 (conceptual disorganization), P3 (hallucinatory behavior), P6 (suspiciousness/ persecution), or G9 (unusual thought content) $>4$, with an absolute increase of 2 on that item since randomization or (b) PANSS item P2, or P3, or P6, or G9 >4, with an absolute increase of 4 on the combined score of these items since randomization. 


\section{Statistical analysis}

AEs were reported by preswitch antipsychotic group separately for the initiation and stabilization phases to address safety concerns during the immediate crossover process after the first AL injection.

Changes from baseline in CGI-S global scale score, PANSS total score, and weight were analyzed using a mixed model for repeated measures (MMRM). The baseline score, study region, visit, and prior medication group were used as factors in the model, and the prior medication group and visit were used as interaction terms. The within-patient variability was modeled using an autoregression 1 covariance matrix. The least-squares mean and standard error at each visit were reported and plotted. For prolactin, the observed value was reported. To assess the statistical significance of mean changes from baseline to all posttreatment visits, a test on the slope by visit, assuming a linear trend, was performed for each preswitch antipsychotic group separately.

\section{Results}

\section{Patients}

Of 242 outpatients who were previously prescribed an oral antipsychotic and enrolled in the 52-week study, 190 (78\%) could be categorized into 1 of 5 preswitch oral groups and were included in this post hoc analysis (Table 1). The other 52 patients were receiving an oral antipsychotic that was either not commonly prescribed or not clearly identified, or the number of patients receiving the oral antipsychotic did not meet the inclusion criteria for the analysis. A summary of preswitch oral antipsychotics taken by patients who were not included in the post hoc analysis is reported in Table
S1. For the purposes of evaluation, patients who previously received risperidone and oral paliperidone were combined into a single group $(\mathrm{n}=52)$. Likewise, patients who previously received high- and mediumpotency conventional antipsychotics were combined into a single "conventional antipsychotic" group $(\mathrm{n}=37)$. Baseline demographic characteristics were similar among the preswitch oral antipsychotic groups (Table 2). Baseline doses of preswitch antipsychotics are summarized in Table S2.

\section{Retention rate: initiation and stabilization phases}

Almost all patients $(96.8 \% ; n=184 / 190)$ received a second AL injection, successfully completing the 4-week initiation phase (Table 1). The likelihood of completing all $3 \mathrm{AL}$ injections over the combined 12 weeks of the initiation and stabilization phases was $92.1 \%(\mathrm{n}=175$ ) 190). Completion rates among the different oral antipsychotic groups for both phases were comparable (Table 1).

\section{Early-onset AEs}

The AEs that occurred in $\geq 2$ patients during the first 12 weeks of switching are reported in Table S3 by preswitch antipsychotic. The overall incidence of akathisia in the initiation phase was $3.2 \% \quad(n=6 / 190)$ : aripiprazole $(3.6 \%)$, risperidone/paliperidone $(3.8 \%)$, quetiapine $(3.8 \%)$, and olanzapine $(5.3 \%)$. No cases of akathisia were reported in the conventional antipsychotic group. The incidence of akathisia was lower in the subsequent 8 weeks (the stabilization phase) $(1.2 \%)$; events were reported only for aripiprazole $(2.0 \%)$ and quetiapine $(4.5 \%)$. One patient discontinued the study because of akathisia over this period. The incidences of

TABLE 1. Patient disposition

\begin{tabular}{|c|c|c|c|c|c|c|}
\hline \multirow[b]{2}{*}{$n(\%)$} & \multicolumn{5}{|c|}{ Preswitch oral antipsychotic } & \multirow[b]{2}{*}{$\begin{array}{c}\text { Total } \\
\mathrm{N}=190\end{array}$} \\
\hline & $\begin{array}{c}\text { ARI } \\
n=56\end{array}$ & $\begin{array}{c}\mathrm{RIS}^{*} \\
\mathrm{n}=52\end{array}$ & $\begin{array}{c}\mathrm{CON}^{\dagger} \\
\mathrm{n}=37\end{array}$ & $\begin{array}{c}\text { QUE } \\
n=26\end{array}$ & $\begin{array}{c}\text { OLA } \\
n=19\end{array}$ & \\
\hline Completed initiation phase ${ }^{\ddagger}$ & $53(94.6)$ & $50(98.1)$ & $37(100)$ & 22 (92.3) & $19(100 \%)$ & $181(95.2)$ \\
\hline Completed initiation/stabilization phase ${ }^{\S}$ & $52(92.9)$ & $47(92.2)$ & $36(97.3)$ & $23(95.8)$ & $17(89.5)$ & $175(92.1)$ \\
\hline \multicolumn{7}{|c|}{ Reasons for discontinuation (over first 12 weeks) } \\
\hline Withdrew consent & $2(3.6)$ & $2(3.8)$ & 0 & $1(3.8)$ & $1(5.3)$ & $6(3.2)$ \\
\hline Adverse events & 0 & $2(3.8)$ & $1(2.7)$ & $1(3.8)$ & $1(5.3)$ & $5(2.6)$ \\
\hline Lost to follow-up & $1(1.8)$ & 0 & 0 & $1(3.8)$ & 0 & $2(1.1)$ \\
\hline Lack of efficacy & 0 & $1(1.9)$ & 0 & 0 & 0 & $1(0.5)$ \\
\hline Protocol deviation & $1(1.8)$ & 0 & 0 & 0 & 0 & $1(0.5)$ \\
\hline
\end{tabular}

\footnotetext{
* Oral paliperidone was grouped with oral risperidone.

${ }^{\dagger}$ Includes medium- and high-potency conventional antipsychotic.

${ }^{\ddagger}$ Received 2 AL injections.

${ }^{\S}$ Received 3 AL injections.

AL, aripiprazole lauroxil; ARI, aripiprazole; CON, conventional; OLA, olanzapine; QUE, quetiapine; RIS, risperidone/paliperidone; SD, standard deviation.
} 
TABLE 2. Baseline characteristics of patients switched from oral antipsychotics to AL

\begin{tabular}{|c|c|c|c|c|c|c|}
\hline \multirow[b]{2}{*}{ Characteristic } & \multicolumn{5}{|c|}{ Preswitch oral antipsychotic } & \multirow[b]{2}{*}{$\begin{array}{c}\text { Total } \\
N=190\end{array}$} \\
\hline & $\begin{array}{c}\text { ARI } \\
n=56\end{array}$ & $\begin{array}{c}\mathrm{RIS}^{*} \\
\mathrm{n}=52\end{array}$ & $\begin{array}{c}\mathrm{CON}^{\dagger} \\
\mathrm{n}=37\end{array}$ & $\begin{array}{c}\text { QUE } \\
n=26\end{array}$ & $\begin{array}{c}\text { OLA } \\
n=19\end{array}$ & \\
\hline Age, years (SD) & $41.6(18.1)$ & $37.3(9.7)$ & $39.4(10.7)$ & $45.0(12.3)$ & $44.2(14.1)$ & $40.7(12.0)$ \\
\hline Male, n (\%) & $28(50.0)$ & $29(55.8)$ & $18(48.6)$ & $17(65.4)$ & $15(78.9)$ & $107(56.3)$ \\
\hline Weight, kg (SD) & $84.0(17.8)$ & $79.8(16.7)$ & $78.0(16.7)$ & $84.4(16.4)$ & $84.0(13.8)$ & $81.7(16.9)$ \\
\hline BMI, $\mathrm{kg} / \mathrm{m}^{2}$ (SD) & $28.4(5.4)$ & $27.6(5.2)$ & $26.9(5.3)$ & $28.9(5.0)$ & $27.5(4.8)$ & $27.9(5.2)$ \\
\hline CGI-S, mean (SD) & $2.8(0.4)$ & $2.8(0.4)$ & $2.7(0.7)$ & $3.0(0.5)$ & $3.0(0.2)$ & $2.8(0.5)$ \\
\hline
\end{tabular}

other AEs of interest were $<6 \%$ in the initiation phase and $<5 \%$ in the stabilization phase (Table 2 ).

The overall incidence of injection site reactions (ISRs) over 12 weeks was $8.4 \%(n=16 / 190)$. The incidence of ISRs was $5.8 \%(n=11 / 190)$ with the first injection, $4.5 \%$ $(\mathrm{n}=8 / 176)$ with the second injection, and $4.1 \%(\mathrm{n}=$ $7 / 169$ ) with the third injection. Most ISRs were reported as injection site pain $(87.5 \% ; n=14 / 16)$.

All groups experienced modest weight changes from baseline in the initiation phase: aripiprazole $(0.35 \mathrm{~kg})$, risperidone/paliperidone $(0.22 \mathrm{~kg})$, conventional $(0.28 \mathrm{~kg})$, quetiapine $(-0.14 \mathrm{~kg})$, and olanzapine $(-0.14 \mathrm{~kg})$; and in the stabilization phase: aripiprazole $(0.33 \mathrm{~kg})$, risperidone/ paliperidone $(0.47 \mathrm{~kg})$, conventional $(0.61 \mathrm{~kg})$, quetiapine $(-0.20 \mathrm{~kg})$, and olanzapine $(-0.28 \mathrm{~kg})$.

Changes in serum prolactin levels from baseline to week 12 by previous oral antipsychotic were assessed for both sexes (Figure S2). Patients in the aripiprazole and quetiapine preswitch groups showed minimal changes in prolactin levels, while those in the conventional, risperidone/paliperidone, and olanzapine groups exhibited greater changes in serum prolactin levels for both sexes. Changes in prolactin levels for males from study entry to week 12 were as follows: aripiprazole $(-1.8 \mathrm{ng} / \mathrm{mL})$, risperidone/paliperidone $(-10.7 \mathrm{ng} / \mathrm{mL})$, conventional $(-14.2 \mathrm{ng} / \mathrm{mL})$, quetiapine $(-4.3 \mathrm{ng} / \mathrm{mL})$, and olanzapine $(-8.1 \mathrm{ng} / \mathrm{mL})$. Changes in prolactin levels for females from study entry to week 12 were as follows: aripiprazole $(-13.0 \mathrm{ng} / \mathrm{mL})$, risperidone/paliperidone $(-37.6 \mathrm{ng} / \mathrm{mL})$, conventional $(-14.0 \mathrm{ng} / \mathrm{mL})$, quetiapine $(-0.8 \mathrm{ng} / \mathrm{mL})$, and olanzapine $(-16.0 \mathrm{ng} / \mathrm{mL})$. Female patients in the risperidone preswitch group experienced significant reduction in prolactin plasma levels $(P=.007)$.

\section{Psychiatric symptoms}

During the initiation and stabilization phases, mean PANSS total scores remained stable across preswitch antipsychotic groups (Figure 2). A test for trend showed

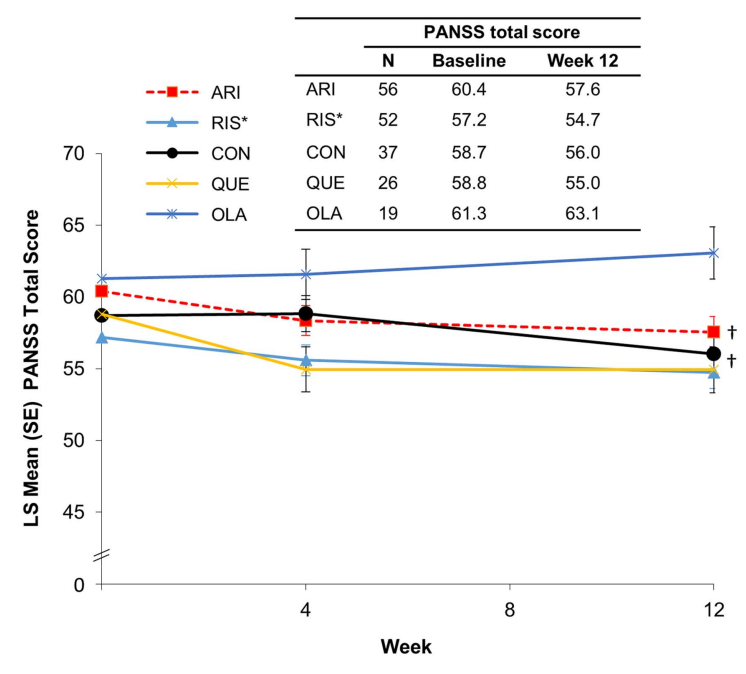

FIGURE 2. PANSS total score by preswitch antipsychotic group (MMRM). *Oral paliperidone was grouped with oral risperidone.

${ }^{\dagger} A$ test for trend showed significant improvement in PANSS total score in patients who received treatment with oral aripiprazole $(P<.03)$ or oral conventional antipsychotics $(P<.04)$ before switching to AL.

ARI, aripiprazole; CON, conventional; LS, least squares; MMRM, mixed model for repeated measures; OLA, olanzapine; PANSS, Positive and Negative Symptom Scale; QUE, quetiapine; RIS, risperidone/paliperidone,

$\mathrm{SE}$, standard error.

significant improvement in PANSS total score in patients who received treatment with oral aripiprazole $(P<.03)$ or oral conventional antipsychotics $(P<.04)$ before switching to AL. Two and three patients relapsed or met the impending relapse criteria during the initiation and stabilization phases, respectively.

\section{Discussion}

There is ample literature to provide guidance on switching between oral antipsychotics, but published information is lacking on switching from oral antipsychotics to longacting formulations. Consequently, many clinicians are 
experienced with switching between oral antipsychotics, but might be hesitant to recommend a change to a longacting formulation because of uncertainty or unfamiliarity. ${ }^{7}$

The main finding of this analysis was that switching patients with relatively stable schizophrenia who were taking an oral antipsychotic to AL is feasible on an outpatient basis. These results provide evidence that the crossover process can be finished by the time the patient returns for a second injection, and that the likelihood of completing the first 12 weeks of the regimen is high $(92.1 \%)$.

Many of the switching outcomes involve the likelihood of completing the switch and the frequency and severity of newly emergent problems that might complicate the immediate switch period. Although some early-onset AEs were likely associated with the change in medication regimen, no unexpected safety events were reported during the initiation or stabilization phases. Treatmentemergent akathisia was infrequent, occurring in only $3.2 \%$ of patients between the first and second $\mathrm{AL}$ injections, with only 1 akathisia-related discontinuation during the 12-week switching period.

We had hypothesized that tolerability issues during the transition to $\mathrm{AL}$, especially during the initiation phase, would be influenced by previous oral antipsychotic use. Results of other "switch" studies showed that the likelihood or magnitude of new issues with tolerability is affected by the specific tolerability profile of the preswitch antipsychotic. ${ }^{12-16}$ In this post hoc analysis, we did not observe any clear association between the early-onset tolerability problems and the patient's previous oral antipsychotic. No relationship was apparent between specific preswitch oral antipsychotics and the likelihood of akathisia or early discontinuation. Only patients who were switched from a conventional antipsychotic to AL did not experience akathisia in the first 4 weeks; however, it is difficult to determine the significance of this observation because of the low overall incidence of akathisia.

It was expected that during the stabilization phase (comprising a period of 2 additional AL injections over 8 weeks without further exposure to oral antipsychotic) any potential issues resulting from withdrawal of the previous antipsychotic, or transient but distressing AEs from the new medication, would have manifested or resolved.

The overall pattern of schizophrenia symptoms during the initiation and stabilization phases was comparable among switch groups, and no trend for symptom exacerbation or significant improvement was noted. The exception was the switch from oral aripiprazole or conventional antipsychotics to $\mathrm{AL}$, in which a trend toward significant improvement in PANSS total score over 12 weeks was observed. It is important to note that the patient cohort was very stable at enrollment and the PANSS total scores changed by $<3$ points. Study limitations include the post hoc nature of the analysis, the small sample sizes in each preswitch antipsychotic group, and the fact that the study was not designed a priori as a switch study.

Potential limitations of the present analysis include its post hoc nature, because the dataset originated from a 1-year, open-label, safety extension study that was not designed to analyze oral switching strategies. In addition, the requirement for patients to be clinically stable at baseline (PANSS total score $>70$ ), together with a frequent study visit schedule, may have contributed to the high retention rate in the 12-week period assessed in the present analysis.

The decision to switch antipsychotic medications should be based on clinical reasons that merit changing antipsychotics in patients who are stable on their current medication regimen. ${ }^{1,5}$ Physicians must assess whether any short-term complications will be balanced by the increased efficacy, safety, and tolerability expected with the new treatment. ${ }^{17}$ Therefore, the possible benefits of switching to a drug associated with fewer adverse metabolic effects must be weighed against the potential risk for the clinical instability associated with changing of treatment. ${ }^{18}$ Switching from a medication associated with a high risk for metabolic problems to one with a lower risk for metabolic complications is a reasonable clinical option, if close monitoring is available. ${ }^{18}$

\section{Conclusion}

The current post hoc analysis demonstrated that switching stable outpatients with schizophrenia from a firstline oral antipsychotic to AL was associated with a high (>90\%) completion rate. The safety and early-onset AEs did not seem to be influenced by the specific oral antipsychotic prescribed before starting the AL regimen. These findings support the feasibility and safety of switching stable outpatients with schizophrenia who are prescribed an oral antipsychotic to AL.

\section{Supplementary material}

To view supplementary material for this article, please visit https://doi.org/10.1017/S1092852918000986

\section{Disclosures}

Peter Weiden, Arielle Stanford, and Yangchun Du are employees of Alkermes, Inc. Chih-Chin Liu is a former employee of Alkermes, Inc.

\section{REFERENCES:}

1. Hasan A, Falkai P, Wobrock T, et al. World Federation of Societies of Biological Psychiatry (WFSBP) guidelines for biological treatment of schizophrenia, part 2: update 2012 on the long-term treatment of schizophrenia and management of antipsychotic-induced side effects. World J Biol Psychiatry. 2013; 14(1): 2-44. 
2. Perkins DO. Predictors of noncompliance in patients with schizophrenia. J Clin Psychiatry. 2002; 63(12): 1121-1128.

3. Quach PL, Mors O, Christensen TO, et al. Predictors of poor adherence to medication among patients with first-episode schizophrenia-spectrum disorder. Early Interv Psychiatry. 2009; 3 (1): 66-74.

4. Weiden PJ, Kozma C, Grogg A, Locklear J. Partial compliance and risk of rehospitalization among California Medicaid patients with schizophrenia. Psychiatr Serv. 2004; 55(8): 886891.

5. Weiden PJ. Switching antipsychotic medications: not enough, too often, or just right? Am J Psychiatry. 2011; 168(9): 882884.

6. Das AK, Malik A, Haddad PM. A qualitative study of the attitudes of patients in an early intervention service towards antipsychotic long-acting injections. Ther Adv Psychopharmacol. 2014; 4(5): 179185.

7. Weiden PJ, Roma RS, Velligan DI, Alphs L, DiChiara M, Davidson B. The challenge of offering long-acting antipsychotic therapies: a preliminary discourse analysis of psychiatrist recommendations for injectable therapy to patients with schizophrenia. J Clin Psychiatry. 2015; 76(6): 684-690.

8. Cruz MP. Aripiprazole lauroxil (Aristada): an extended-release, long-acting injection for the treatment of schizophrenia. P T. 2016; 41(9): 556-559.

9. Meltzer HY, Risinger R, Nasrallah HA, et al. A randomized, doubleblind, placebo-controlled trial of aripiprazole lauroxil in acute exacerbation of schizophrenia. J Clin Psychiatry. 2015; 76(8): 1085-1090.

10. Nasrallah HA AR, Du Y, Stanford AD, Claxton A, Weiden PJ. Longterm safety and tolerability of aripiprazole lauroxil in patients with schizophrenia. CNS Spectrums. 2018; In Press.
11. Alkermes Inc. Aristada (Aripiprazole Lauroxil) Prescribing Information. 2017; https://www.aristadahcp.com/downloadables/ ARISTADA-PI.pdf. Accessed May 2017.

12. Weiden PJ, Simpson GM, Potkin SG, O'Sullivan RL. Effectiveness of switching to ziprasidone for stable but symptomatic outpatients with schizophrenia. J Clin Psychiatry. 2003; 64(5): 580-588.

13. Newcomer JW, Campos JA, Marcus RN, et al. A multicenter, randomized, double-blind study of the effects of aripiprazole in overweight subjects with schizophrenia or schizoaffective disorder switched from olanzapine. J Clin Psychiatry. 2008; 69(7): 1046-1056.

14. Casey DE, Carson WH, Saha AR, et al. Switching patients to aripiprazole from other antipsychotic agents: a multicenter randomized study. Psychopharmacology (Berl). 2003; 166(4): 391-399.

15. McEvoy JP, Citrome L, Hernandez D, et al. Effectiveness of lurasidone in patients with schizophrenia or schizoaffective disorder switched from other antipsychotics: a randomized, 6-week, openlabel study. J Clin Psychiatry. 2013; 74(2): 170-179.

16. Weiden PJ, Citrome L, Alva G, et al. A trial evaluating gradual- or immediate-switch strategies from risperidone, olanzapine, or aripiprazole to iloperidone in patients with schizophrenia. Schizophr Res.. 2014; 153(1-3): 160-168.

17. Newcomer JW, Weiden PJ, Buchanan RW. Switching antipsychotic medications to reduce adverse event burden in schizophrenia: establishing evidence-based practice. J Clin Psychiatry. 2013; 74 (11): 1108-1120.

18. Stroup TS, McEvoy JP, Ring KD, et al. A randomized trial examining the effectiveness of switching from olanzapine, quetiapine, or risperidone to aripiprazole to reduce metabolic risk: comparison of antipsychotics for metabolic problems (CAMP). Am J Psychiatry. $2011 ; 168(9)$ : 947-956. 